\title{
Design and Analysis of BER Rate For 5G Network Using OFDM
}

\author{
Suraj R. Wailthare ${ }^{1}$, Rupali Dasarwar ${ }^{2}$, Pratibha Motwani $^{3}$ \\ ${ }^{l} P G$ Student, ${ }^{2,3}$ Asst. professor \\ Wainganga college of engineering and management, Nagpur, India, 440012.
}

DOI: 10.46335/IJIES.2020.5.10.3

\begin{abstract}
The fifth telecommunication generation (5G) is expected to be deployed in 2020 with significant improvement and services. This paper studies a preliminary model of Indonesia $5 G$ channels based on statistical spatial channel model (SSCM) for operating frequency of $28 \mathrm{GHz}$. We calculate the estimate channel capacities of Indonesia $5 G$ (per link) from the obtained power delay profile (PDP) to observe the outage probability representing the theoretical performances of $5 G$ in Indonesia. The outage probability is calculated from the probability when the channel capacity $C$ dropped into a value below the channel coding rate $R$ with a probability of $P(R \geq C)$ due to the dynamic fading, indicating a failure of detection.
\end{abstract}

Keywords-Bit Error Rate(BER),Orthogonal frequency division multiplexing(OFDM), Chaotic channel.

\section{I- INTRODUCTION}

$\mathbf{T}_{\mathrm{he}}$ fifth telecommunication generation $(5 \mathrm{G})$ is expected to be realized in 2020 after the second phase release of the $5 \mathrm{G}$ standard. Implementation in Indonesia requires well preparation of infrastructure, especially the parameter design based on the channel model. Channel models are important part of wireless communications systems, since the capacity (per-link) is depending on the channels [1]. Mathematical calculations combined with communication theory, can be used to predict the performances of $5 \mathrm{G}$ networks of several locations via the channel models measured at several cities in Indonesia. There are several channel models proposed for communications, for example, the Okumura-Hata Model in [2] for Okumura measurement and for Hata model, an empirical formulation based on Okumura model. Okumura-Hata model This research is in part supported by the Telkom University Scientific Research Grant of "Hibah Penelitian Internasional 2017-2018" on PolarRaptor Codes-Structured Super-Dense Networks for The Internet-of-Things (IoT)" and by Ristek Dikti on "5G-POINT: Preparing Optimal 5G Indonesia Networks", 2018-2019. 0102030405060708090 100 Frequency $(\mathrm{GHz}) 00510 \quad 1520 \quad 25 \quad 30 \quad 35 \quad 40 \quad 45$ Attenuation $(\mathrm{dB})$ at $200 \mathrm{~m}$. Attenuation at frequency candidates of $5 \mathrm{G}$ from $1 \mathrm{GHz}-100 \mathrm{GHz}$ simulated by combining dry air, vapor, haze, and rain in Indonesia measured at distance of $200 \mathrm{~m}$ from the transmitter using NYUSIM simulator. is intended for large scale. However, the considered model in this paper is a model of small scale fading. Furthermore, Okumura-Hata model is a model which is valid for frequency from 150 $\mathrm{MHz}$ to $1500 \mathrm{MHz}$, while $5 \mathrm{G}$ is expected to operate in frequency range between $1 \mathrm{GHz}$ to $100 \mathrm{GHz}$. Each frequency range has special characteristics, especially for very high frequency of $5 \mathrm{G}$ since the attenuation is also high. We measure the attenuation of Bandung city using a model developed by New York University (NYUSIM) under nonline-of-sight (NLOS), taking over hundreds channel realizations as shown in. We capture attenuattion $(\mathrm{dB})$ at 200 meters for frequency range of 1$100 \mathrm{GHz}$. We can observe from Fig. 1 that each frequency has different attenuation due to different effect of environmental parameters such as temperature, humidity, and air pressure also confirmed that a sudden high attenuation in Bandung city also happens at $60 \mathrm{GHz}$ similar to the environment of USA as shown in $3[7,5]$ 
Removal.The considered structure of CP-OFDM with simple channel coding to confirm the theoretical outage performance of $5 \mathrm{G}$ in Indonesia. Since each country has different environmental parameters, we need a channel model of $5 \mathrm{G}$ developed independently to achieve best performances. In this paper, we consider NYUSIM using statistical spatial channel model (SSCM) technique to model the channel from measurements. SSCM is considered in this paper since SSCM measures the channel with large number of samples and analyze statistically by taking into account the spatial characteristic of the environment including types of antenna. The channel model has been used and effective in performance evaluation, for example, in based on real field experiment in Germany. The calculation of outage probability has been demonstrated in and which agree with the result of practical coding, even though the outage performances are calculated independently.

\section{II- METHODOLOGY}

Implementation of FBMC OFDM In the proposed work, a new method FBMC-OFDM is implemented by using a filter at the transmitter and receiver. The implication of the proposed work is that, FBMC-OFDM subcarriers are divided into even and odd blocks and filtration are applied on it, which is still not viewed in any former study. It enhances the throughput of the system by scaling down the parameters reason for the internal interference of the system. SIMULATION RESULTS The simulated results are obtained and analysed by using a MAT-LAB simulation discussed below. The simulation parameters are given in Parameters of Simulation This shows the subcarriers of OFDM, FBMC and FBMC OFDM with overlapping factor ( $\mathrm{k}=1$ and 2$)$.. Subcarriers of OFDM, FBMC and FBMC OFDM The effect of Carrier Frequency Offset (CFO) is shown by Figure 2. The above figure states that for smaller CFO, the performance of BER is good as compared to higher value of CFO.As shown above, For $\mathrm{CFO}=0.1$, the performance is better as compared to $\mathrm{CFO}=0.2$ and 0.3 . OFDM of BER vs Eb/No with CFO OFDM FBMC FBMC OFDM FFT Size $=64$ Subcarriers $=64 \mathrm{CP}=11 \%$ loss insertion. Filter $=$ No used FFT Size $=64$ Subcarriers $=64 \mathrm{CP}=$ No $\mathrm{CP}$ Filter $=$ PHYDAS Overlapping Factor $\mathrm{K}=2$ Length of Filter $=\mathrm{K} * \mathrm{M}-1$ FFT Size $=64$ Subcarriers $=64 \mathrm{CP}=11 \%$ loss Filter $=$ PHYDAS Overlapping Factor $\mathrm{K}=2$ Length of Filter = K*M-1 32 Unauthenticated Download Date | 10/24/17 2:37 PM Scientific Bulletin of the Electrical Engineering.

\section{III- DESIGN}

The advantage of the OFDM is its ability to use an overlapping subcarrier for modulating the parallel data streams, thereby making the technique more effective than the traditional technique as far as bandwidth efficiency is concerned. To avoid the related intercarrier interference, the subcarriers used must be orthogonal. Fast Fourier transform is used to drive the needed set of orthogonal subcarriers. In OFDM, the low-rate data streams are modulated into the subcarriers to ensure flat fading in each of the subcarrier used. OFDM is a simple method that is used in various applications, especially in unit modulation in which the data transmitted occupies the entire bandwidth available. An important point to note is that the OFDM data are modulated via narrowed subcarriers that have a bandwidth smaller than the bandwidth of the channel, thereby leading to the development of a flat fading condition in each of the subcarriers used. Besides, the cyclic prefix (CP) is used after every symbol of the subcarrier. The $\mathrm{CP}$ represents the tail samples, with the LCP as its length. The presence of these symbols improves the channel flatness in each of the subcarriers, thereby enhancing the probability of selecting the channel frequency. Coding schemes such as conventional codes are very important in the digital domain; they are used to collect and code the binary input data bits. The coded bit stream is then interleaved to enhance diversity gain after which the bits are put together and mapped using the matching points in the constellation. The complex data is serialized.

Therefore, the known mapping schemes are mapped together with known pilot symbols to obtain the modulated data mentioned in the IFFT operator is then performed on the parallel complex data after serialization has been applied to the parallel converter. Again, the transformed data is put together according to the number of required subcarriers for transmission. In every block of data, the CP is inserted in accordance to the system specification after which the data is multiplexed in a serial order. At this level, the data is modulated with OFDM and is ready for transmission. To convert the digital data to analog, a digital-to-analog converter is used. Next, RF modulation is performed, and the signal is converted to a transportable frequency. In the event it is transmitted using the antenna, the OFDM signals overcome the challenges of the wireless network channel. 


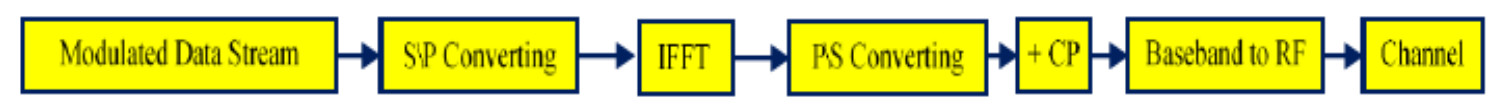

Fig-OFDM transmitter

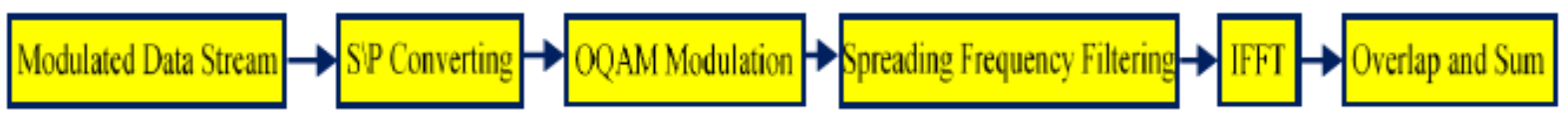

Fig-FBMC transmitter

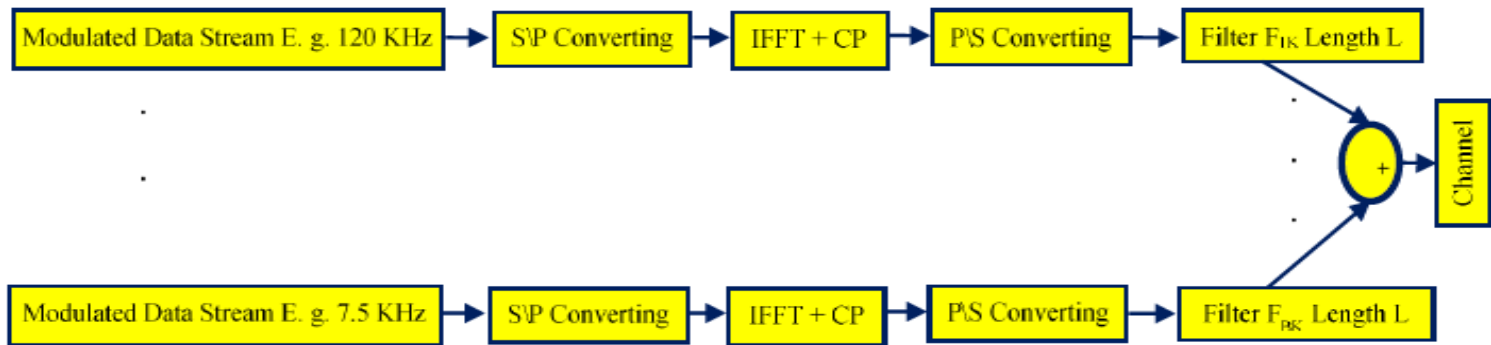

Fig. 4. F-OFDM transmitter scheme

\section{III- CONCLUSION}

This study investigated two major candidates for $5 \mathrm{G}$ systems, namely, F-OFDM and FBMC. A detailed comparison of these candidate waveforms with the conventional CP-OFDM waveform was performed. Through simulation, it has been confirmed that each of the waveforms has positive and negative impacts on various parameters. In this regard, we have noted that other criteria need to be considered to select the perfect waveform generation. We considered the tail issues, latency, complexity, and many other factors. Both FOFDM and FBMC waveforms improve the power spectral density but affect the PAPR ratio negatively. At the same time, the F-OFDM has been found to improve the BER rate. We conclude our study by asserting that FOFDM is a better candidate for $5 \mathrm{G}$ networks when compared to other schemes investigated in this work.

\section{ACKNOWLEDGMENT}

This publication is a result of acknowledge the sincere efforts and valuable time given by the professors of electronics branch of Wainganga college of engineering and management, who provided insight and expertise that greatly assisted the research. I would like to express my special thanks of gratitude to my teacher as well as our principal who also helped me to do this wonderful project, which also help me in doing lot of research and I came to know about so many new things. I am really thankful to them.

\section{REFERENCES}

[1] Ramadhan AJ. Overview and implementation of the two most important candidate $5 G$ waveforms. J. Theor. Appl. Inf. Technol., Accepted, 2019.

[2] Ramadhan AJ. Implementation of 5G FBMC PHYDYAS Prototype Filter. Int. J. Appl. Eng. Res. 2017;12(23):13476-13481.

[3] Sahin A, Guvenc I, Arslan H. A Survey on Multicarrier Communications: Prototype Filters, Lattice Structures, and Implementation Aspects. IEEE Commun. Surveys Tutorials 2014;16(3):1312-1338.

[4] Banelli P, Buzzi S, Colavolpe G, Modenini A, Rusek F, Ugolini A. Modulation Formats and Waveforms for $5 G$ Networks: Who will be the Heir of OFDM? An Overview of Alternative Modulation Schemes for Improved Spectral Efficiency. IEEE Signal Process. Mag. 2014;31(6):80-93.

[5] Metis Project. Metis deliverable D1.1: Scenarios, requirements and KPIs for $5 G$ mobile and wireless systems.

https://cordis.europa.eu/docs/projects/cnect/9/317669 1080/deliverables/001-METISD11v1pdf.pdf, 2013. 
[6] Bogucka H, Kryszkiewicz, P, Jiang T, Kliks A. Dynamic Spectrum Aggregation for Future $5 G$ Communications. IEEE Commun. Mag. 2015;53(5):35-43.

[7] Wunder G, Jung P, Kasparick M, Wild T, Schaich F, Chen Y, Ten Brink S, Gaspar I, Michailow N, Festag A, Mendes LL. 5GNOW: Non-orthogonal, Asynchronous Waveforms for Future Mobile Applications. IEEE Commun. Mag. 2014;52:97-105.

[8] Feichtinger H, Strohmer T. Gabor Analysis and Algorithms: Theory and Applications. Springer Science \& Business Media, 1998.

[9] Le Floch BL, Alard M, Berrou C. Coded Orthogonal Frequency Division Multiplex. In Proc. IEEE 1985;83:982-996. 